\title{
NEW OBSERVATIONS ALLOWING THE DIFFERENTIATION OF LATE ASEXUAL STAGES OF CYSTOISOSPORA CANIS FROM DEVELOPING MICROGAMONTS IN THE INTESTINES OF EXPERIMENTALLY INFECTED DOGS
}

\author{
J. P. Dubey ${ }^{1}$ and D. S. Lindsay ${ }^{2}$ \\ ${ }^{1}$ United States Department of Agriculture, Agricultural Research Service, Beltsville Agricultural Research Center, Animal Parasitic Diseases Laboratory, \\ Beltsville, Maryland, 20705-2350. \\ 2 Department of Biomedical Science and Pathology, Virginia Maryland College of Veterinary Medicine, Center for One Health Research, Virginia Tech, 1410 \\ Prices Fork Road, Blacksburg, Virginia 24061. \\ Correspondence should be sent to J. P. Dubey at: jitender.dubey@ars.usda.gov
}

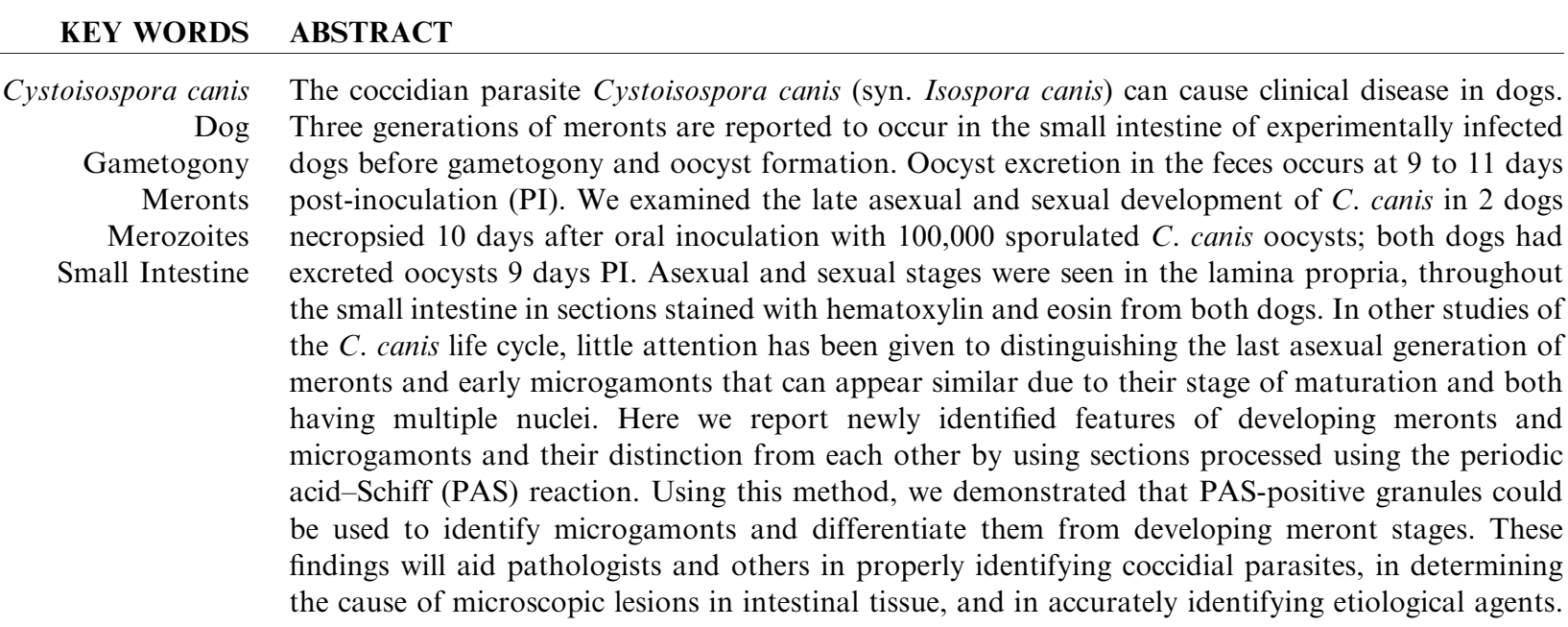

Cystoisospora canis (syn. Isospora canis) is a coccidian that can cause clinical disease in dogs (Mitchell et al., 2007). Lepp and Todd (1974) described the endogenous development of C. canis, using oocysts of a cloned culture as inoculum, in coccidia-free dogs inoculated orally with 150,000 sporulated oocysts. One dog was necropsied daily from 1 to 12 days post-inoculation (PI). They reported 3 generations of meronts occurring 5-8 days PI in the lamina propria of the distal one-third of the villi in the small intestine. They documented their findings using line drawings and micrographs of intestinal tissue sections stained using Heidenhain's iron hematoxylin or Harris's hematoxylin and eosin (HE) (Lepp and Todd, 1974). They based their descriptions on what was considered a standard life cycle for Eimeria species that have a set number of generations of meronts (multinucleate stage) that produce merozoites (single nucleus stage); the last generation of merozoites produces microgamonts (multinucleate stage) and macrogamonts (single nucleus stage) with oocysts (single nucleus stage) occurring after fertilizalization of macrogamonts by microgametes produced by microgamonts. They (Lepp and Todd, 1974) depicted up to 19 nuclei in line drawings of first-generation meronts. They did not include micrographs or line drawings of multinucleate second-generation meronts but depicted a parasitophorous vacuole containing 3 immature third-generation meronts with 8,7 , and 4 nuclei (fig. 11 of Lepp and Todd, 1974). This leaves much unknown regarding the structure of these stages and can lead to misinterpretation. These authors (Lepp and Todd, 1974) reported that gamonts were seen 7-10 days PI, but no mention was made of asexual or sexual stages in the dogs examined 11 and 12 days PI. The culture of oocysts used by Lepp and Todd (1974) has been discontinued, and there are no archived histological sections for re-evaluation.

Recently Dubey and Lindsay (2019) re-evaluated asexual development of $C$. canis in 2 dogs necropsied 10 days after oral inoculation with 100,000 C. canis oocysts; both dogs had excreted oocysts 9 days PI. Asexual and sexual stages were seen in the lamina propria, throughout the small intestine in sections stained with HE. The results of that study indicated that there are many asexual generations, and they agreed that early stages of microgamonts could not readily be distinguished from meronts. In the present paper, we report in more detail the development of 


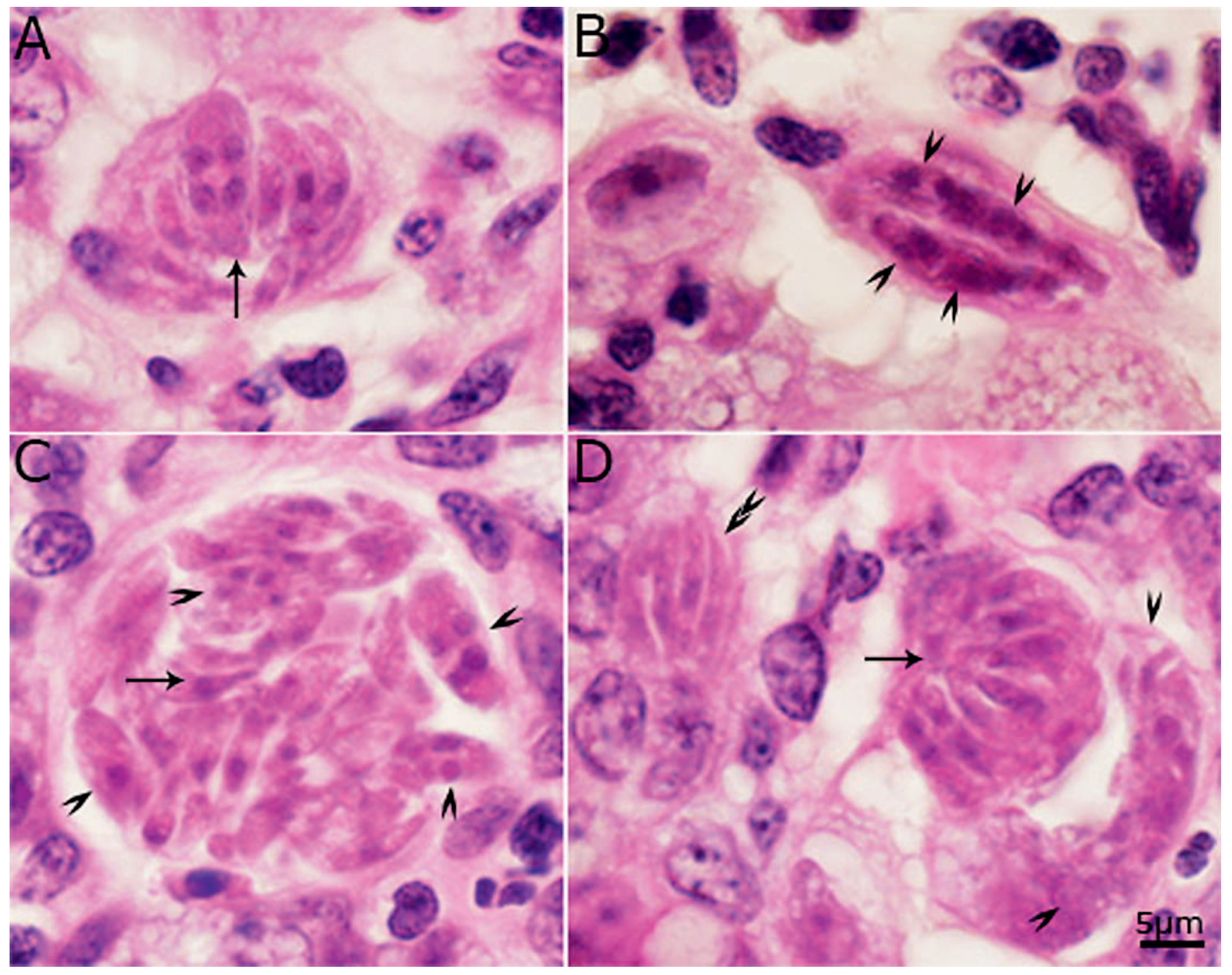

Figure 1. Asexual stages of Cystoisospora canis in the lamina propria of small intestine of dogs 10 days after oral inoculation with 100,000 oocysts. Hematoxylin and eosin stain. Bar applies to all figures. Different shapes and sizes of meronts. (A) Meront with mature merozoites and a merozoiteshaped organism with 4 nuclei (arrow). (B) Meront with 2 elongated merozoite-shaped-meronts with multiple nuclei (arrowheads). (C) Large meront with mature merozoites (arrow) and several multinucleated meronts (arrowheads) in the same vacuole. (D) Three meronts. Meront on the left (double arrowheads) has 4 elongated merozoites. The meront in the center has merozoites arranged like a rosette. Arrow points to a merozoite nucleus giving the appearance of a residual body. The meront on the right (opposing arrowheads) is irregular-shaped (arrowheads) and has developing merozoites. Color version available online.

microgamonts and macrogamonts of $C$. canis and their distinction from asexual stages in the small intestines of dogs. This is needed because distinguishing the last asexual generation of meronts and early microgamonts, which can appear similar due to their stage of maturation and both having multiple nuclei, is needed to correctly identify coccidial parasites in tissues and to aid in determining the identity of the parasites present. The correct identification of infectious agents is always a priority for those studying the epidemiology and transmission of infectious diseases.

The tissue sections examined in this study were from the experiments reported by Mitchell et al. (2007). The dogs were female beagles and obtained commercially (Covance, Cumberland, Virginia) at $6 \mathrm{wk}$ of age. Feces of these dogs were examined by sugar flotation for 3 days before inoculation with sporulated $C$. canis oocysts. Coccidian oocysts were never detected in feces of these dogs before inoculation (Mitchell et al., 2007). The 2 dogs (AJV and AIZ2) were each inoculated orally with 100,000 sporulated oocysts and euthanatized 10 days later. The study was approved by the Institutional Animal Care and Use Committee of Virginia Tech, Blacksburg, Virginia. The dogs excreted $C$. canis oocysts with a prepatent period of 9 days. Loops of duodenum, jejunum, and ileum were fixed in $10 \%$ neutral buffered formalin and processed for histological sections. Sections were examined after staining with $\mathrm{HE}$ as described previously (Dubey and Lindsay, 2019). Additionally, for the present study sections were examined after staining with periodic acid-Schiff (PAS) reaction, counter-stained with hematoxylin (PASH). In total 91 segments stained with HE or PASH were examined at $\times 1,000$ magnification in an Olympus $\mathrm{AX} 70$ microscope and 


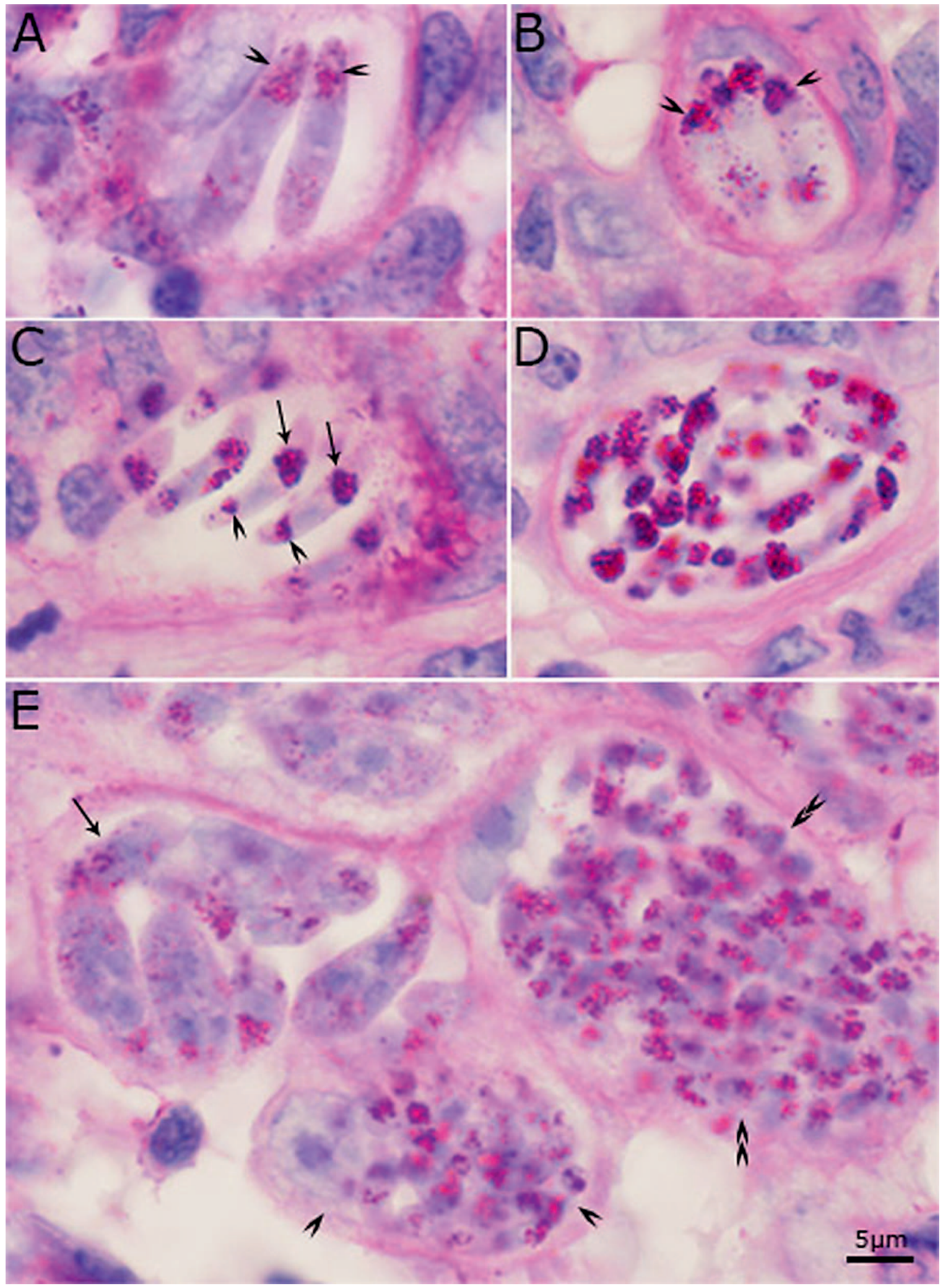

Figure 2. Asexual stages of Cystoisospora canis in the lamina propria of small intestine of dogs 10 days after oral inoculation with 100,000 oocysts. Periodic acid-Schiff (PAS) reaction, counter-stained with hematoxylin (PASH). All stages are PAS-positive. Bar applies to all figures. (A) Two elongate merozoite-shaped meronts in a vacuole. Arrowheads point to polar PAS-positive granules. (B) Four merozoite-shaped meronts in vacuole. The PASpositive granules are concentrated at poles (arrowheads), apparently more at the pointed ends. (C) Meront with mature merozoites. Note distribution of large (arrows) and small (arrowheads) PAS-positive granules. (D) Mature meront with intensely PAS-positive granules. (E) Three meronts with varying degree of PAS-positive merozoites and meronts. Developing meront containing multinucleated merozoite-shaped merozoites (arrow), small meront (arrowhead), and a large meront (double arrowheads). Color version available online. 


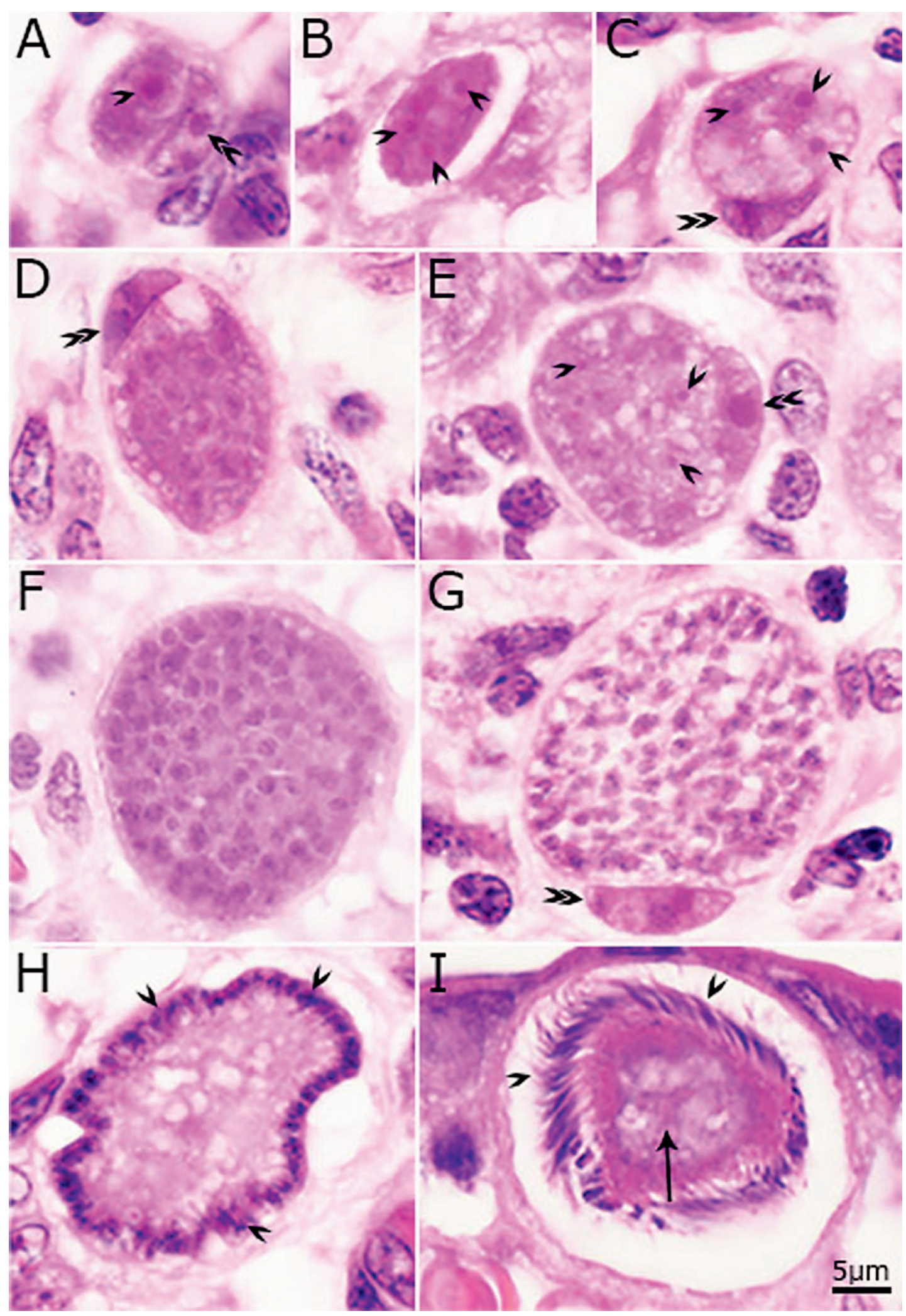

Figure 3. Microgamonts of Cystoisospora canis in the lamina propria of small intestine of dogs 10 days after oral inoculation with 100,000 oocysts. Hematoxylin and eosin stain. Bar applies to all figures. Double arrowheads point to host cell nucleus on all figures. (A, B) Early microgamonts with 3 or more nuclei (arrowheads). (C-E) Ovoidal microgamonts with 3 or more vesicular nuclei. (F) Numerous $(>80)$ vesicular nuclei filling the entire microgamont. (G) Microgamont with compact nuclei. (H) Developing microgamont with peripherally arranged spherical nuclei (arrowheads). (I) Numerous flattened microgametes with compact nuclei (arrowheads) with whip-like tails around a large residual body (arrow). Color version available online. 


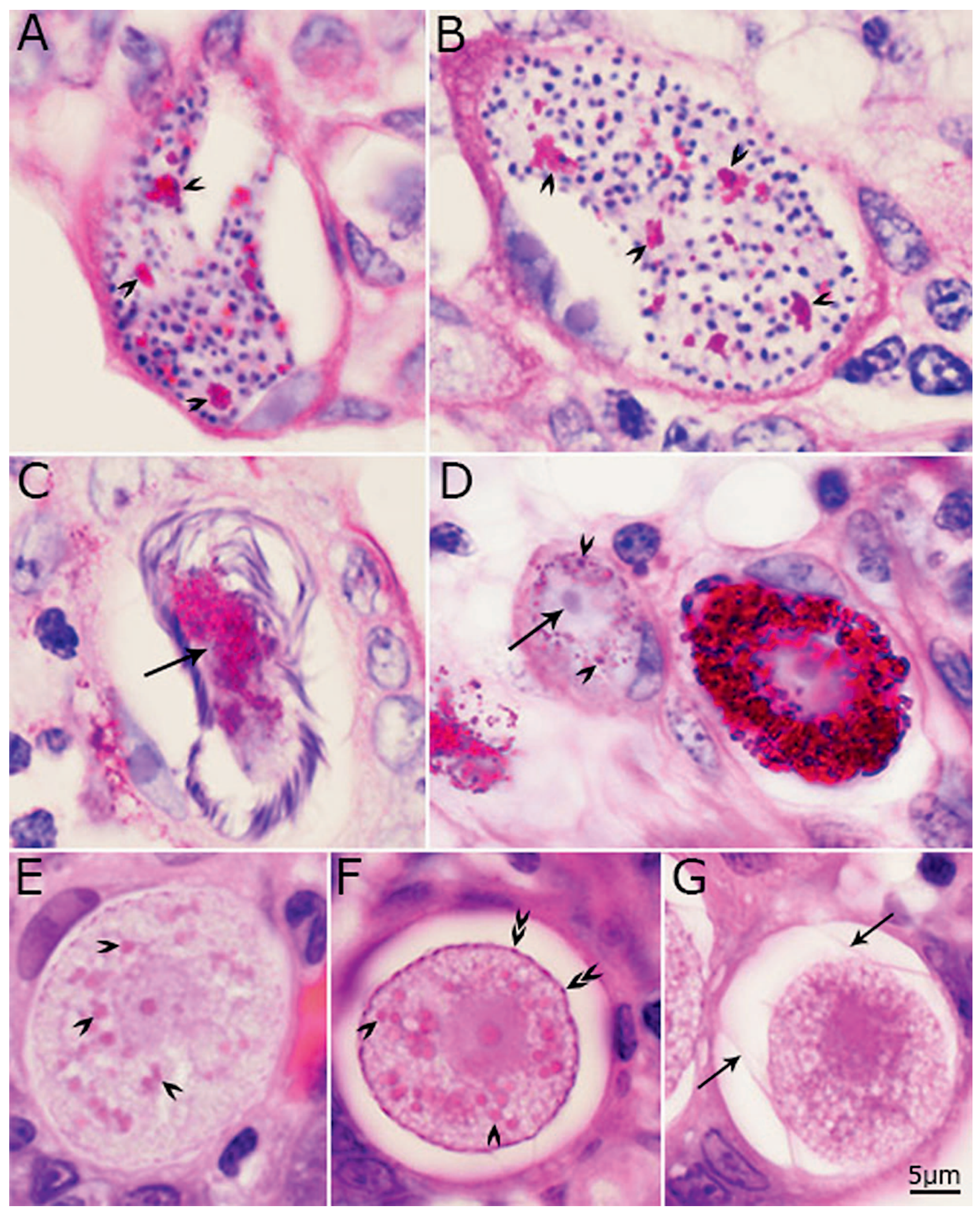

Figure 4. Gamonts of Cystoisospora canis in the lamina propria of small intestine of dogs 10 days after oral inoculation with 100,000 oocysts. (A-D) periodic acid-Schiff (PAS) reaction, counter-stained with hematoxylin, (E-G) hematoxylin and eosin stain. Bar applies to all figures. (A, B) Immature microgamonts with many compact nuclei and PAS-positive residual bodies (arrowheads). (C) Mature microgamont with a central residual body containing a PAS-positive accumulation of granules (arrow). (D) Early macrogamont (arrow) with a large nucleus and prominent nucleolus surrounded with PAS-positive granules adjacent to a more developed intensely PAS-positive (arrowhead) macrogamont. (E, F) Macrogamonts each with a large nucleus and nucleolus. Arrowheads point to eosinophilic bodies (wall-forming bodies [WFBs]). A row of peripheral granules is present (double arrowheads) in gamont in F. (G) Oocyst with absence of WFBs. Arrows point to crumpled oocyst wall. Color version available online. 
stages photographed using a DP73 camera (Olympus, America Inc., Melville, New York).

Asexual and sexual stages were seen in the lamina propria, throughout the small intestine. In HE-stained sections, it was difficult to distinguish early microgamonts from meronts (multinucleate stages preceding evidence of merozoite formation). However, it was possible to distinguish them with PASH staining. Different sizes and shapes of meronts were found (Figs. 1, 2). Conventional meronts containing many nuclei were not detected. The maximum number of nuclei in a meront was 8 , and early meronts were merozoite-shaped. The PAS positivity varied with the stage of merozoite development. Mature merozoites were more intensely PAS-positive than the early stages (Fig. 2). Two discrete areas of PAS-positive granules were seen: the granules in the center of the merozoite were more intensely stained than those at the polar ends (Fig. 2C). A residual body was absent in meronts.

Gamonts were seen throughout the small intestine. The early developmental stages of microgamonts were PAS-negative. The earliest microgamonts were ovoidal to pear-shaped with 2 or more vesicular nuclei (Fig. 3A-C). As karyokinesis proceeded, the nuclei became smaller but remained vesicular even in microgamonts with numerous nuclei that occupied the entire gamont (Fig. 3F). One or more PAS-positive granules of different sizes were seen in the cytoplasm of microgamonts (Fig. 4A, B). Eventually the nuclei transformed from being vesicular to being compact, remained spherical, and moved to the surface of the gamont (Fig. 3E-H). Sometimes invaginations of the surface membrane were observed. Microgametogenesis was monocentric. The single nucleus of a microgamete was densely basophilic, somewhat flattened with whip-like eosinophilic lashes (likely flagella) extending into the parasitophorous vacuole (Fig. 3I). Mature microgametes were arranged at the periphery of a large residual body that was slightly basophilic in the center but eosinophilic at the surface beneath microgamete nuclei in $\mathrm{HE}$ sections (Fig. 3I).

Early macrogamonts could be distinguished from microgamonts by the presence of a large central nucleus and prominent nucleolus in macrogamonts. Even the earliest macrogamont contained PAS-positive granules (Fig. 4D), whereas early microgamonts were PAS-negative. The PAS-positive intensity and variable sizes of PAS-positive granule material were present in macrogamonts. This increased as the macrogamont increased in size (Fig. 4D). In sections stained with HE, lightly eosinophilic wall-forming bodies (WFBs; syn., plastic granules of Lepp and Todd, 1974) were seen in macrogamonts (Fig. 4E, F). These WFB structures were not seen in oocysts (Fig. 4G). The oocyst wall was membrane-like and faintly eosinophilic in mature unsporulated oocysts in HE sections (Fig. 4G).

The results reported here provide a detailed and complete description of gametogony of $C$. canis and supplement the description of late asexual stages and microgamonts provided by Lepp and Todd (1974). In addition, we elaborated on the development of macrogamonts. For example, Lepp and Todd (1974) did not find WFBs, which they termed plastic granules in macrogamonts that are described here. The macrogamonts of the Sarcocystidae do not have the densely eosinophilic wall-forming bodies found in many of the Eimeriidae, and this is likely why they did not report their presence in macrogamonts. Ultrastructural studies are needed to describe the divisional process in gamonts and meronts of $C$. canis to better understand the developmental biology of this group of coccidians in relation to other Sarcocystidae and Eimeriidae.

Descriptions presented in this study will aid researchers in human and veterinary medicine to recognize the developmental stages of mammalian intestinal coccidia. This can often be a difficult task because intestinal coccidia are variable in appearance and location in a host. It will provide these scientists with a starting point to make logical decisions to pursue an accurate etiological diagnosis.

We would like to thank Camila Cezar and Meghan Sadler for assistance. The study examined archived material. The authors report no conflicts of interest.

\section{LITERATURE CITED}

Dubey, J. P., And D. S. Lindsay. 2019. Re-evaluation of asynchronous asexual development of Cystoisospora canis in intestines of dogs. Journal of Parasitology 105: 25-26.

LEPP, D. L., AND K. S. TodD. 1974. Life cycle of Isospora canis Nemeséri, 1959 in the dog. Journal of Protozoology 21: 199206.

Mitchell, S. M., A. M. Zajac, S. Charles, R. B. Duncan, and D. S. Lindsay. 2007. Cystoisospora canis Nemeséri, 1959 (syn. Isospora canis), infections in dogs: Clinical signs, pathogenesis, and reproducible clinical disease in beagle dogs fed oocysts. Journal of Parasitology 93: 345-352. 